\title{
Laboreal
}

Volume $16 \mathrm{~N}^{\circ} 1 \mid 2020$

Quando o trabalho real é tabu

\section{2-2020: ¿Se liberará, por fin, Quebec del amianto?}

2012-2020: irá o Quebeque, finalmente, libertar-se do amianto?

2012-2020: le Québec va-t-il enfin sortir de l'amiante?

2012-2020: will Quebec, at last, set free from asbestos?

\section{Micheline Marier}

Traductor. Fernanda Romero

\section{OpenEdition}

\section{Journals}

\section{Edición electrónica}

URL: http://journals.openedition.org/laboreal/16242

DOI: 10.4000/laboreal. 16242

ISSN: $1646-5237$

Editor

Universidade do Porto

Referencia electrónica

Micheline Marier, «2012-2020: ¿Se liberará, por fin, Quebec del amianto?», Laboreal [En línea], Volume $16 N^{0} 1$ | 2020, Publicado el 01 julio 2020, consultado el 15 septiembre 2020. URL : http:// journals.openedition.org/laboreal/16242

Este documento fue generado automáticamente el 15 septiembre 2020

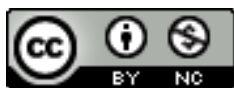

Laboreal está licenciado com uma Licença Creative Commons - Atribuição-NãoComercial 4.0 Internacional. 


\title{
2012-2020: ¿Se liberará, por fin, Quebec del amianto?
}

\author{
2012-2020: irá o Quebeque, finalmente, libertar-se do amianto? \\ 2012-2020: le Québec va-t-il enfin sortir de l'amiante? \\ 2012-2020: will Quebec, at last, set free from asbestos?
}

\section{Micheline Marier}

Tradución : Fernanda Romero

\section{NOTA DEL EDITOR}

Traducción: Fernanda Romero -fernandaromero.trad@gmail.com

1 Durante 136 años, se extrajo amianto del suelo de Quebec que era después exportado para todo el mundo. Producíamos mucho más de lo que conseguíamos consumir y hoy nos enfrentamos a un proyecto de explotación para fines comerciales de los residuos que nos dejaron esas décadas. ¿Será mejor "volver a colocarlos en el agujero" o cubrirlos con vegetación, si eso fuera posible? Para los habitantes de Asbestos y de Thetford Mines, cuyas ciudades crecieron gracias al oro blanco desde que su explotación empezó, en 1876, hasta terminar, en septiembre de 2012, es difícil borrar casi un siglo y medio de estrecha vinculación entre mineros, empresas mineras, ciudades de amianto y gobiernos. Retrocedamos en el tiempo para entender la situación actual.

\section{1876-1949 - De la fiebre del oro blanco hasta la denuncia de la asbestosis}

Desde 1877, se han abierto cerca de veinte minas en la región de Thetford Mines y, en 1879, se inició la explotación de un yacimiento de amianto en Asbestos que nacía, así, como la población del oro blanco de Quebec. Fue ahí entonces cuando industriales británicos y estadounidenses se apresuraron a comprar minas. En 1925, la industria era 
constituida por cinco grandes protagonistas: dos empresas mineras canadienses que exportaban la producción y tres fábricas norteamericanas que allí se abastecían de materia prima (Vallières, 1989).

Los primeros registros de que hay conocimiento sobre enfermedades de los trabajadores expuestos al amianto surgieron en el inicio del siglo XX. Con escasas excepciones, las publicaciones sobre la asbestosis (o amiantosis) se refieren a los trabajadores de la industria transformadora y no a los mineros. En los Estados Unidos, las empresas de amianto, ya con demandas judiciales de los trabajadores contra ellas, conocían los riesgos añadidos de cáncer porque financiaban la investigación, pero ocultaban los resultados tanto de los trabajadores como de los clientes (McCulloch, 2005).

4 En Quebec, hacía varias décadas que las ciudades de amianto se preocupaban con los polvos (Cinq-Mars, 1994). Sin embargo, poco o nada podían hacer porque eran dependientes de las empresas de extracción del mineral.

5 La sindicalización de los mineros de amianto aumentó sobre todo a partir del 1936 y para su federación, asociada a la futura Confederación de los Sindicatos Nacionales (CSN), la asbestosis constituía ya una preocupación (Dumont, 1956). Cuando, en el 1949, la federación organizaba una gran huelga suscitada por cuestiones salariales y de gestión, el diario de Montreal Le Devoir publicó un reportaje sobre la catástrofe sanitaria de la asbestosis en la región. El tema de la eliminación de los polvos vino, así, a sumarse a las reivindicaciones de los huelguistas.

6 La huelga en Asbestos, que duró 4 meses y medio y que fue salvajemente reprimida después de que Johns-Manville recurriera a esquiroles, terminó en un desorden público y la policía provincial fue llamada a intervenir. Durante cinco días reinó un clima de terror en la cuidad. La huelga terminó sin haberse abordado el tema de los polvos (Delisle \& Malouf, 2004).

\section{1950-1972 - La preocupación latente con los polvos}

7 En la década de 1950 empezó un periodo de restructuración de este sector de la industria con la llegada de dos grandes empresas norteamericanas: en 1952, ASARCO, en la rama de la industria transformadora y una filial de General Dynamics que, en 1968, compró la única industria extractora canadiense que todavía existía en esa época (Vallières, 1989; Parent, 1981).

8 A partir de ese momento, toda la industria de esta rama era extranjera y "no industrializante" (Parent, 1981, p. 2), ya que tan solo se transformaba entre el 2 y el 2,5\% de la producción en Quebec. Y, en 1969, aunque el 95,9\% de los trabajadores eran francófonos, el idioma de trabajo de las cumbres era el inglés (AMAQ, 1971), un hecho inaceptable en un momento en que emergía un doble nacionalismo, el quebequés y el canadiense, centrado en el control de los recursos naturales nacionales (Parent, 1981).

9 Tanto en Thetford Mines como en Asbestos, la apertura de nuevos pozos condujo a la relocalización de barrios enteros. Después de décadas intentando, sin éxito, diversificar la economía, los responsables políticos locales querían ser ellos mismos a ocuparse del ordenamiento y desarrollo económico. 
10 Mientras tanto, empezaron a surgir otras preocupaciones: salud en el trabajo, temas medioambientales, contestación del proceso productivo. La década de 1950 había empezado con el final decepcionante de la huelga de 1949. Después de ese, la industria minera había incurrido en gastos considerables para eliminar los polvos de las minas y del proceso de molido del amianto. Pero el aumento de la producción, no obstante, condujo a una situación insostenible.

11 En el plan de la salud en el trabajo, se establecieron normas de exposición al amianto: en 1968, en Gran Bretaña, y en 1971, en los Estados Unidos, donde había acabado de ser promulgada una ley sobre salud y seguridad en el trabajo. En Quebec, el ministerio de los Recursos Naturales empezó a aplicar, en 1950, el valor límite de exposición de la American Conference of Governmental Industrial Hygienists (ACGIH), pero todavía no había una norma oficial.

12 Además, en 1966, la industria creó el Instituto de Medicina del Trabajo y del Ambiente de Montreal (Institut de médecine du travail et de l'environnement de Montréal, IUMTE) vinculado a la Universidad McGill, como reacción a las declaraciones del Dr. Irving Selikoff sobre la relación estadística entre el amianto y el cáncer en los montadores de aislamientos térmicos en los Estados Unidos. Fue aprobado un proyecto de investigación del profesor Corbett McDonald sobre los peligros del amianto y, en 1970, él mismo declaró en Thetford Mines que, con los niveles de exposición a los polvos entonces existentes, no habría probablemente ningún riesgo significativo de cáncer de pulmón (McDonald, 1970). Pese a esto, los trabajadores del amianto, la mayor fuerza sindical de la región, dieron inicio a una lucha por la salud en el trabajo.

\section{1973-1982 - El control del amianto y la protección de la salud de los mineros}

13 EN 1973, el amianto se convirtió en el símbolo de la espoliación de los recursos de Quebec y de la irresponsabilidad de las empresas mineras en materia de salud de los trabajadores y de las comunidades (Chartier \& Thiboutot, 1974). El recién creado Parti québécois señaló el carácter no renovable de la riqueza colectiva que representaba el subsuelo de Quebec (Parti québécois, 1973). Se plantearon entonces dos cuestiones: saber si sería necesario nacionalizar la industria del amianto para reasumir su control y si, para proteger la salud de los trabajadores, se debía imponer el uso controlado de este mineral.

En el periodo de 1970-1974, se alcanzó el pico de la producción y de las exportaciones de amianto, pero la victoria electoral del Parti québécois, en 1976, introdujo cambios significativos en la industria. La Sociedad Nacional del Amianto (Société nationale de l'amiante, SNA), fundada poco tiempo después, tendría como objetivo producir y vender fibras y productos de amianto $\mathrm{y}$, al mismo tiempo, realizar investigaciones científicas para desarrollar nuevos productos y nuevos métodos. EN 1980, la SNA nacionalizó las instalaciones de Turner \& Newall, abrió fábricas de transformación y se asoció a empresas ya existentes. En 1982, concluyó el proceso de adquisición de la sociedad minera que quince años antes había sido comprada por General Dynamics (Vallières, 1989). En ese mismo año, Johns-Manville, propietaria de la mina de Asbestos, se declaró en quiebra a raíz de los miles de demandas de trabajadores víctimas interpuestas en los Estados Unidos (Gagnon, 2004). Un grupo de antiguos directivos de la empresa compró 
la mina sin, no obstante, dominar los mercados de Johns-Manville para dar salida a su producción.

La Confederación de los Sindicatos Nacionales (CSN), que representaba a la gran mayoría de los mineros, realizó, en 1971, un estudio económico sobre la importancia del amianto para Quebec y otro sobre las enfermedades profesionales causadas por el amianto (Wassef, 1974), asociando, entonces, el equipo del Dr. Selikoff.

En marzo de 1975, los trabajadores del amianto de la región de Thetford Mines iniciaron una huelga. Los metalúrgicos, afiliados en la Federación de los Trabajadores de Quebec (FTQ), pasaron a representar a los trabajadores de dos minas de la región, formando un frente común con la CSN para presentar, de este modo, las mismas reivindicaciones en materia de empleo y salarios. Fue en ese momento que los resultados del estudio llevado a cabo por el equipo del Dr. Selikoff se hicieron públicos: casi el $60 \%$ de los trabajadores examinados tenían los pulmones afectados por el amianto (CSN, 1975). A partir de entonces, las reivindicaciones en materia de salud en el trabajo pasarían a constar en las negociaciones de la CSN y, poco después, los metalúrgicos adoptarían la misma posición ( $\mathrm{FTQ}, 1975)$. Los trabajadores de Asbestos, que se habían separado de la CSN para crear la Central de los Sindicatos Democráticos (Centrale des syndicats démocratiques, CSD), no participarían en esa huelga.

17 La huelga terminó después de siete meses y medio. Suscitó varias acciones del gobierno de Quebec contra la asbestosis, entre las cuales la creación de una comisión para analizar las cuestiones de salubridad en la industria del amianto. Dicha comisión (Comité Beaudry) terminó sus trabajos en finales de octubre de 1976. La CSN, la FTQ y la CSD, bien como los Trabajadores Unidos de la Industria Automovilística (Travailleurs unis de l'automobile, TUA) presentaron propuestas muy sólidas cuyo análisis el Comité, en gran parte, apoyó. Así, recomendó, en particular, la adopción de una ley sobre salud y seguridad que previese una amplia participación de los trabajadores. El entonces muy reciente gobierno del Parti québécois, se comprometería a aplicar plenamente esas recomendaciones.

Esto ha constituido una importante victoria para los sindicatos, una vez que consiguieron que la sociedad quebequesa reconociera no solo la dimensión del problema causado por los polvos del amianto, sino también la posibilidad de controlarlo más eficazmente. Para ellos, el problema no estaba en el amianto en sí mismo, pero en la "ignorancia, en la carrera hacia las ganancias y en los tabúes" (Rodrigue, 1976).

19 Sin embargo, poco después la lucha de los trabajadores del amianto se convirtió también en una lucha desesperada por el mantenimiento de sus empleos. La entrada en los años 1980 trajo una profunda caída en la producción y en el empleo a raíz de la crisis de $1982 \mathrm{y}$, posteriormente, sobre todo debido al movimiento por la prohibición del amianto iniciado por Dinamarca en 1972 (IBAS, 2016).

\section{1983-2012 - Deterioro de la industria del amianto y lucha por la manutención del empleo}

20 En 1983, se abrió una nueva era. Los directivos quebequeses francófonos se quedaron, a partir de entonces, al frente de la industria, aunque con los puestos de trabajo reducidos a poco más de mitad con relación a 1979 (CSN, 1983). 
21 En la escena internacional, el movimiento por la prohibición fue creciendo. En los Estados Unidos, la Agencia de Protección Ambiental (EPA) intentó, en 1983, prohibir el amianto. En 1996, la prohibición en Francia hizo temer un efecto dominó. En 2006, la OIT, que en junio de 1986 había aprobado el Convenio n.ำ 162, optando por la utilización controlada del amianto crisotilo, fue evolucionado, es decir, en particular el BIT en conjunto con la OMS, para una posición a favor de la prohibición total del amianto (o asbesto).

Frente a esto, los gobiernos de Canadá y de Quebec decidieron actuar en defensa de la industria, interviniendo ante los organismos internacionales y enviando misiones al Norte de África, Sudamérica y Asia para promocionar el amianto de Quebec. En 2002, el gobierno de Quebec, con el fin de demostrar, a nivel internacional, su buena fe en la defensa del uso controlado del amianto crisotilo, adopta incluso una política para ampliar la utilización de ese mineral en obras públicas. Además, en las reuniones de la Conferencia de las Partes en el Convenio de Róterdam [ $\left.{ }^{1}\right]$, Canadá se opone a la inclusión del amianto crisotilo en el Anexo III, evitando así que las exportaciones de ese producto sean sometidas al procedimiento PIC (consentimiento fundamentado previo) como producto peligroso.

Pero la crisis continuó y, en 2012, el nuevo gobierno del Parti québécois se negó a seguir apoyando la industria del amianto. Fue el fin de la explotación del amianto en Quebec.

Todavía, para los sindicatos de los mineros, los treinta años que habían entonces transcurrido desde 1983 estuvieron particularmente marcados por la lucha para salvar a la industria donde trabajaban y a sus regiones.

De hecho, ya en 1976, los metalúrgicos se habían opuesto a los sindicatos escandinavos y neerlandeses que intentaban lograr que la Federación Internacional de los Trabajadores de las Industrias Metalúrgicas (FIOM) se asociara a la lucha por la prohibición del amianto (Gérin-Lajoie, 1982). Pero la CSN y la CDS no se pararon y, en 1983, las tres centrales sindicales se unieron a los demás actores socioeconómicos de las dos regiones del amianto y crearon una comisión de revalorización del amianto (Courrier Frontenac, 1989). Buscaron, de este modo, defender el amianto en la escena nacional, pero también a nivel internacional, en particular en los países en vías de desarrollo, defendiendo las ventajas de las tuberías (o tubos) de fibrocemento, más baratas que las demás y que irían permitir "llevar agua potable e instalar saneamiento a millones de personas (Gérin-Lajoie, 2016). Desafortunadamente, desvalorizaron las dificultades de la aplicación de un control eficaz del uso del amianto en esos países. Por cierto, ni siquiera en Quebec se lograría eso en las fábricas y en el sector de la construcción.

No obstante, la opinión pública empezó a cambiar en Quebec. El Instituto Nacional de Salud Pública de Quebec (INSPQ), creado en 1998, constató, en 2003, que el 23\% de la industria transformadora del amianto en Quebec superaba los valores límite de exposición exigidos por la ley (Simard, 2003). Por otro lado, un número cada vez más alto de científicos, sindicalistas y médicos canadienses venían a público a denunciar las políticas canadienses y quebequesas y exigir la prohibición del amianto. Y, en 2011, la CSN ha exigido entonces el fin de la explotación y de la utilización del amianto (CSN, 2011). De ahí que una de las primeras medidas adoptadas por el nuevo gobierno de 2012 del Parti québécois, haya sido la de acabar con la explotación minera del amianto en Quebec. 
27 Fue, todavía, necesario esperar hasta finales de 2016 para que el gobierno federal decidiese prohibir la utilización del amianto en Canadá, con efecto a partir del 30 de diciembre de 2018. Sin embargo, esa ley consagró (además de algunas excepciones relacionadas, concretamente, con la industria del cloro y de la soda caustica, las instalaciones nucleares y equipos militares) una importante ambigüedad en lo que respecta a los residuos mineros: impide que se vendan para la construcción civil y la gestión del paisaje, a menos que la provincia donde se desarrollan esas actividades autorice esa utilización (Gouvernement du Canada, 2018).

\section{2020: ¿otras formas de utilizar el oro blanco?}

En efecto, durante la década de 1980, surgieron varias propuestas para recuperar los compuestos minerales existentes en los residuos de amianto. Por consiguiente, en el inicio de la década de 2000, la entrada en funcionamiento de una fábrica de producción de magnesio trajo una bocanada de esperanza a Asbestos, aunque unos años más tarde dicha esperanza fue rápidamente ahogada por la producción más barata de la industria china.

29 A pesar de ello, otros proyectos están hoy renaciendo. Se mantiene, no obstante, la cuestión de saber si será posible extraer residuos de las escorias y transformarlos industrialmente sin volver a poner en peligro a los trabajadores de esas fábricas y poblaciones circundantes. Las autoridades sanitarias públicas temen que no. Sin embargo, del lado de los empresarios, muchos se oponen a la reducción del valor límite de exposición al amianto impuesto por la ley de Quebec - aunque sea diez veces superior al de otros países - para que la implementación de proyectos en esa área de la recuperación de residuos no salga perjudicada.

La Oficina de Audiencias Públicas sobre el Medio Ambiente (Bureau d'audiences publiques sur l'environnement) de Quebec tiene que emitir un dictamen hasta finales del mes de julio de 2020. ¿Tendrá esta organización de apoyo al gobierno el mismo coraje del Comité Beaudry en 1977? Y si es así, ¿tendrá el actual gobierno el coraje del gobierno de esa época?

31 En verdad, la industria ha dejado detrás suya un ambiente contaminado en esta región, en razón, desde luego, de los innumerables montones de residuos mineros. Por otro lado, el amianto sigue estando presente en muchos edificios habitacionales, industriales y comerciales. Además, hay un número muy alto de trabajadores de la industria transformadora y de la construcción civil, bien como de ocupantes de eses edificios, cuya salud fue gravemente afectada y que tienen enormes dificultades para ser reconocidos como víctimas de esa violencia tóxica.

Queda mucho por hacer para liberar a Quebec del amianto. 


\section{BIBLIOGRAFÍA}

AMAQ (1971). Le producteur d'amiante, Montréal : AMAQ (juillet/août).

Chartier, J., \& Thiboutot, S. (1974). L'amiante en '73. Sherbrooke, CRDE (revista de imprensa).

Cinq-Mars, F. (1994). Thetford Mines à ciel ouvert : histoire d'une ville minière, 1892-1992. Thetford Mines : Ville de Thetford Mines.

Courrier Frontenac, 29 avril 1989, p.A-5.

CSN (1975). Travailleurs de l'amiante - Région de Thetford. Montréal : CSN (Le travail, mars).

CSN (1983). Tract syndical CSN. Montréal, Archives de la CSN.

CSN (2011). Service des communications de la CSN. L'amiante au-delà des mines. Montréal : CSN (mars).

Delisle, E. \& Malouf, P.K. (2004). Le Quatuor d'Asbestos. Autour de la grève de l'amiante. Montréal : Varia. https://doi.org/10.7202/1055070ar

Dumont, D. (1956/1970). Histoire du syndicalisme. In P.E. Trudeau (Dir.), 1970, La Grève de l'amiante. Une étape dans la Révolution industrielle au Québec (pp.125-140). Montréal : Les Éditions du Jour.

FTQ (1975). Le Monde ouvrier, № spécial.

Gagnon, J. (2004). Histoires de pêche à la mouche. Trois essais d'histoire politique. Sainte-Foy: Presses de l'Université Laval.

Gérin-Lajoie, J. (1982). Les Métallos - 1936-1981. Montréal : Boréal Express.

Gérin-Lajoie, J. (2016). Entrevue avec Jean Gérin-Lajoie, 30 mars 2016.

Gouvernement du Canada (2018). Règlement interdisant l'amiante et les produits contenant de l'amiante. Ministre de la Justice du Canada : DORS/2018-196. http://lois-laws.justice.gc.ca

IBAS (2016). Chronology of National Asbestos Bans. http://www.ibasecretariat.org/ chron_ban_list.php, revista em fevereiro de 2016 (Página consultada a 3 Março de 2016).

McCulloch, J. (2005). Mining and Mendacity or How to Keep a Toxic Product in the Marketplace. International Journal of Occupational and Environmental Health, 11(4), 398-401. https://doi.org/ 10.1179/oeh.2005.11.4.398

McDonald, J.C. (1970). Étude sur l'amiante et la santé: un rapport intérimaire aux employeurs et employés de l'industrie de l'extraction de l'amiante du Québec. Montréal : Fonds des Métallurgistes d'Amérique (BANQ. 2B.1.374 Bell-7285).

Parent, R. (1981). La Bourgeoisie canadienne et le capital étranger dans le développement de l'industrie de l'amiante au Canada. Thèse de doctorat, Département de Sociologie, Université de Montréal.

Parti québécois (1973). Programme officiel.

Rodrigue, N. (1976). L'amiante ou quosse ça donne 25 ans après. Montréal, CSN.

Simard, R. (2003). L'industrie de la transformation de l'amiante. In INSPQ, Fibres d'amiante dans l'air intérieur et extérieur (pp.33-45). http://www.inspq.qc.ca

Thiboutot, S. (1975). L'amiante en '74, Sherbrooke, CRDE (revista de imprensa).

Vallières, M. (1989). Des Mines et des Hommes : histoire de l'industrie minérale québécoise des origines au début des années 1980. Québec: Publications du Québec. 
Wassef, K. (1974). Bref rapport d'activités et programme d'action dans les mines d'amiante. Montréal : Service de recherche de la CSN, Archives de la CSN.

\section{NOTAS}

1. Ver nota [5] del texto de Annie Thébaud-Mony ("Amianto: una contaminación sin fronteras, sin fin y con total impunidad") en el presente dossier.

\section{AUTORES}

\section{MICHELINE MARIER}

Asociación de Víctimas del Amianto de Quebec michelinemarier@contrechamps.ca 